\title{
Flight Deck Workload and Acceptability of Verbal and Digital Communication Protocols
}

\author{
Summer L. Brandt ${ }^{1}$, Joel Lachter ${ }^{1}$, Arik-Quang V. Dao ${ }^{1}$, Vernol Battiste ${ }^{1}$, \\ and Walter W. Johnson ${ }^{2}$ \\ ${ }^{1}$ San Jose State University, Moffett Field, CA 94035, USA \\ ${ }^{2}$ NASA Ames Research Center, Moffett Field, CA 94035, USA \\ \{summer.1.brandt, joel. lachter, quang.v.dao, \\ vernol.battiste-1, walter.w.johnson\} @nasa.gov
}

\begin{abstract}
The Federal Aviation Administration hopes to convert air traffic management to Trajectory Based Operations (TBO), under which aircraft flight plans are known to computer systems which aid in scheduling and separation. However, few aircraft flying today have equipment designed to support TBO. We conducted a human-in-the-loop simulation of TBO using current fleet equipage. Three aircraft equipage levels were explored: Voice (the equipment currently used), FANS (the Future Aircraft Navigation System datacom designed for use in TBO), and ACARS (a datacom system widely used for communication with Airline Operation Centers). FANS uplinked flight plans can be automatically loaded into the Flight Management System, while ACARS delivers text that must be entered manually. Pilots rated various aspects of the procedures. Voice was preferred to FANS, with ACARS rated worst, apparently because of slow response times for requests with datacom. Using a mixture of Voice and datacom may provide the benefits of both.
\end{abstract}

Keywords: Trajectory Based Operations (TBO), Datacom, NextGen, Humanin-the-Loop simulation.

\section{Introduction}

Air traffic is increasing beyond the capacity of the National Air Space (NAS). To fix this, the United States government developed a plan referred to as the Next Generation Air Transportation System or NextGen [1]. One focus of NextGen is to shift workload away from air traffic control (ATC) by providing automated tools to aid controllers in scheduling and maintaining separation between aircraft. However, in order to do this, the automation must know the aircrafts' future plans, not just their current speed and direction. Under current day operations, if an aircraft encounters weather or traffic, ATC will typically send it on a vector, a change in course in which only a new direction is specified. For NextGen automation to work, a new flight plan will have to be entered into a computer on the ground (typically referred to as the "host") that specifies a complete path reconnecting with the original flight path. Such procedures are part of an operational concept known as Trajectory Based Operations (TBO). TBO poses a challenge for pilot-controller communications because significantly more information must be exchanged between ATC and the flight deck. 
The flight deck needs not only a direction to fly but also the locations of all subsequent waypoints until it connects with the original flight plan (e.g., a precise location to turn off the current flight plan and a precise location to turn back).

In current day operations, updated flight plan information is conveyed by voice communications. Voice communication provides pilots with speed and flexibility in negotiations with controllers. However, because voice information is inherently ephemeral, pilots and controllers frequently request clarifications [2]. Message blocking occurs when more than one message is transmitted simultaneously on the same channel resulting in all but one of the messages being omitted or interrupted when they reach the intended recipient. Message blocking occurs particularly when radio frequencies are congested in areas of the air space where traffic density is highest. Further, communication errors, as well as workload increase with the complexity of the message being transmitted [3]. The more complex TBO messages will likely increase workload, frequency congestion and communication errors. To examine solutions to these communication issues, researchers have implemented and compared digital communication protocols with voice [2].

In addition to voice, most transport category aircraft are also equipped with datacom technology called the Aircraft Communications Addressing and Reporting System (ACARS). This system is currently used for communication between aircraft and their Airline Operations Centers (AOCs) but can be used for the transmission of text-based messages between pilots and controllers. ACARS is not prone to errors due to intelligibility, and aids memory because once transmitted the message is perpetually available to the human operator [3]. One of the main advantages of ACARS over voice is the ability to transmit complex messages accurately. However, data would need to be manually entered into the Flight Management System (FMS) with ACARS making it cumbersome to use, and ultimately making transaction times and workload in some cases comparable to voice protocols [4]. Similarly, for pilots to make requests of ATC using ACARS, they would have to type the message into the $\mathrm{CDU}$, which is noticeably slower than making a voice request. Integrating datacom and the FMS could help reduce these costs.

Such an integrated datacom system is the Future Air Navigation System (FANS). FANS allows flight plans in the FMS to be downlinked as requests and uplinked clearances to be loaded into the FMS. Unfortunately, relatively few planes in the NAS are currently FANS equipped, largely because procedures for FANS equipped aircraft are only used in oceanic environments, and then only for arrivals. Datacom - FANS or ACARS - cannot be exploited until procedures are developed and put in place. Research into using datacom for TBO has focused on implementation of controller clearances. In weather impacted airspace, however, aircraft must also communicate requests to the ground. Further, previous research in TBO environments has focused on aircraft with FANS equipage that (as noted above) is still uncommon in the NAS. Mueller [5], for example, examined the use of FANS for implementing controller clearances. Mueller found that this system was satisfactory to implement trajectorybased clearances from controllers.

In a follow-up study, Mueller and Lozito examined flight deck procedures for trajectory-based clearance negotiations [6]. They compared procedures for handling uplinked strategic trajectory clearances that varied the responsibility distribution 
between the pilot flying and the pilot monitoring (sharing versus non-sharing) in one simulation study and whether to print out datacom clearances in another. All procedures evaluated were rated to be generally suitable.

\section{Current Study}

The current research examines issues related to a near-term implementation of TBO where aircraft are expected to have more or less the same equipage as today. Data were collected on both air traffic controller and pilot participants; however, here we report only pilot data. This study specifically examined the ability of aircraft as currently equipped to maintain TBO while modifying trajectories during weather impacted flights. Weather impacted operations were selected in order to require twoway communication between pilots and controllers: Pilots make requests for weather and may reject controller clearances if they take them into weather; controllers issue clearances for traffic and may reject aircraft requests if they take them into traffic. We examined trajectory change requests and clearances using three types of aircraft equipage: Voice only, ACARS and FANS.

\section{Method}

\subsection{Participants}

Participants were sixteen commercial airline pilots with glass cockpit experience (eight per week) and four retired TRACON controllers (two per week) recruited from similar simulations who were familiar with the prototype NextGen ATC tools used in this simulation. Pilots were divided into two person crews with the more experienced chosen as the captain. They remained together for the duration of their participation.

\subsection{Equipment}

Four dual-pilot stations and two controller stations were operated by study participants. Confederates operated a simulation manager station, two pseudo-pilot stations that handled additional non-experimental aircraft and two ghost controller stations that handled aircraft after they left the sector.

Dual Pilot Stations. Each dual-pilot station consisted of three monitors, on which the controls and displays necessary for operating a generic Boeing transport category aircraft were simulated. An autopilot Mode Control Panel (MCP) allowed direct manipulation of the heading, speed and altitude of the aircraft and dual Control Display Units (CDU's) allowed manipulation of the flight plan in the FMS. Flight path and navigation information was presented on dual Cockpit Situation Displays (CSD's) and dual Primary Flight Displays (PFD's). Controls for hand flying the aircraft (e.g., yoke) were not available.

The autopilot MCP, CDUs, PFDs and alerting display were driven by MACS, the Multi-Aircraft Control System developed by the Airspace Operations Lab at NASA Ames [7]. These operated as on most Boeing transport category aircraft. The CSD was 
developed by NASA Ames Flight Deck Display Research Laboratory [8] and presented a 2D display of navigation information, weather radar targets and TCASlike traffic.

\subsection{General Procedures}

The study was run in two one-week sessions. Each week began with one day devoted to training, three days scheduled for data collection and a final day for make-up trials and debriefing. The entire simulation consisted of thirty-two 20-minute scenarios.

During each trial two simulation worlds were run simultaneously, each world containing one participant controller and two participant dual-pilot flight decks. Background aircraft flown by pseudo-pilots provided a realistic traffic load of approximately 16 aircraft at any time.

Once a minute, pilots used a touch-screen computer to rate their workload on a 1 to 5 scale. After each trial pilots gave overall workload and flight acceptability ratings, again using a 1 to 5 scale. A post-simulation questionnaire asked pilots to use a 5point Likert scale to rate items related to concept acceptability, safety and procedures, and simulation realism. Pilots were provided space to add comments.

\subsection{Scenarios}

All experimental flight decks were arrivals into Louisville International Airport (SDF), reaching top of descent near the eastern edge of the sector. Pilots flew west to east through the sector and negotiated a storm front on the eastern side of the sector.

There were four starting conditions at the beginning of the scenario as defined by the location of the weather and traffic. The weather for each of these four starting conditions evolved in various ways creating 16 total scenarios. Because the weather evolved differently from trial to trial, neither the controllers nor the pilots could create a path through the weather until they watched it develop.

\subsection{Experimental Design}

The experimental design had three fixed factors, Airspace Mixture, Aircraft Equipage and Pilot Role (Pilot Flying vs Pilot Monitoring). There were also three random factors, Scenario, Crew and Controller.

Aircraft Equipage specified the datacom communication capability of the individual participant aircraft: FANS, ACARS and Voice. The Airspace Mixture factor, which specified the proportion of FANS, ACARS, and Voice aircraft in the sector, was only relevant to controller workload and performance and will not be further considered in this report.

Each aircrew was run in a total of 32 scenarios, 12 FANS equipped, 12 Voice equipped, and 8 ACARS equipped. Each aircrew saw each of the 16 weather system/traffic patterns twice. In the trials using these repeated scenarios the aircrew always flew different aircraft (approaching the weather system from a different angle) and had a different Aircraft Equipage type. 


\subsection{Communications Procedures}

Procedures had to be developed for pilots to request and implement trajectory modifications. Procedures were initially developed in which proposed flight plan amendments developed by ATC included an initial waypoint on the current route two minutes ahead of the aircraft. This was added to allow time to negotiate, implement, and possibly reject the proposal before any modification began. However, during training and initial runs it became apparent that, for Voice aircraft, communicating this added waypoint increased controller workload beyond tolerable limits. As a result, the procedures were modified so that, for Voice aircraft, pilots proposed and controllers constructed maneuvers that were off the nose; if necessary controllers then amended the flight plan in the host to be consistent with the path the aircraft was flying (e.g., when there was a significant delay between the time the controller entered the flight plan into the host and when the flight deck implemented the initial turn). In addition, the initial maneuver for downlinked requests from FANS-equipped aircraft were also always off the nose of the aircraft. This is the result of a limitation in FANS automation. Proposed ground automation pushes an initial maneuver point along two minutes in front of ownship during the development of a trajectory modification; current FANS implementation does not do this. Manually inserting an initial waypoint that does not move in this manner encounters a number of problems (e.g., the aircraft is not equipped to calculate the location of such a point).

\section{Flight Deck Procedures}

\section{Voice Aircraft}

Procedures on the flight deck for Voice aircraft were similar to those followed today, except that trajectory-based requests and clearances were required. Voice clearances typically took the form of adding a waypoint (e.g., "ABC123, direct HILLS then PRINC remainder of the route unchanged"). Pilots then entered this new routing into their FMS. Pilots encountering weather could request a deviation direct a named waypoint or a new track. Controllers then entered a new flight plan based on this request into the host computer on the ground and issued the appropriate clearance.

\section{FANS Equipped Aircraft}

When an uplinked clearance was received on a FANS equipped aircraft, a message appeared on the alerting display on the flight deck. The procedure for the pilot-notflying was to navigate to the ATC Messages page on the CDU, load the clearance into the FMS, and then, if acceptable, send an accept message via the CDU and execute the flight plan. Otherwise, the pilot would reject the clearance and follow up with a revised request to ATC via datacom or voice. For requests, pilots developed a modified flight plan in the CDU using standard tools, downlinked it to ATC, and then monitored the message status to see if it was accepted or rejected. Accepted requests could be executed. Rejected requests were typically followed up with a proposed clearance from ATC. As noted above, although FANS equipped, ATC uplinks always included an initial modified waypoint approximately two minutes in front of the aircraft. FANS trajectory request downlinks were always for initial turns off the nose of the aircraft. 


\section{ACARS Equipped Aircraft}

Automation translated ATC clearances for ACARS aircraft into an uplinked free text message that appeared on the ACARS menu in the CDU. As with FANS messages, these were announced on the message alerting display. The pilot-not-flying would then navigate to the ACARS messages page on the CDU, and then read and confirm it with the flying pilot before entering it into the FMS.

Clearances were presented in two parts. First, the clearance itself, which contained the path an aircraft was to fly listed as a series of waypoints (possibly including latitude-longitude coordinates). Second, an "FMS contingency" procedure to be executed if the crew could not enter the clearance before arriving at the first modified waypoint, initially located approximately two minutes ahead of the aircraft. The FMS contingency provided the predicted time at which an aircraft would reach this first waypoint, and a track the pilots should fly from the first to the second waypoint. Pilots were taught to initially enter the second waypoint into the CDU. They could then execute the new flight plan at the contingency time and remain on their flight plan if they did not have time to enter the first waypoint. In practice this became the standard as the first waypoint was almost always a lat/long and onerous to enter.

ACARS aircraft accepted/rejected clearances by free texting back WWW for Wilco, and UNA for Unable, which were interpreted appropriately by the automation. The pilots were trained to use the free text function of the ACARS ATC message page when making trajectory requests by ACARS, which controllers would read and turn into amendments that were then uplinked back to the aircraft.

\section{Results}

Repeated-measures ANOVAs were used to analyze the pilot ratings. The p-values were adjusted using Greenhouse-Geisser for violations of Sphericity where appropriate. Unless otherwise stated, all ratings were averaged by crew.

\subsection{Crew Workload Ratings}

At the end of each trial, pilots rated their overall and peak workload ( 1 none to 5 unmanageable) associated with their flight and with weather (see Table 1). Across all four workload measures, crews consistently rated their workload highest in the ACARS condition and lowest in the Voice condition. These differences were significant for overall workload associated with the flight $(F(2,14)=9.75, p<.01)$ and peak workload associated with the flight $(F(2,14)=5.83, p<.05)$. Overall and peak workload associated with avoiding weather $(F(2,14)=4.40, p=.07 ; F(2,14)=$ $3.13, p=.08$, respectively) were not significant but followed a similar pattern.

Workload probes were also collected at one-minute intervals throughout each trial. Missing data (due to computer error) occurred $1.3 \%$ of the time. There were no significant differences with missing data by Aircraft Equipage, $(F<1)$.

Individual pilot response latencies to the workload probes were then analyzed as a function of Pilot Role and Aircraft Equipage. The effect of Aircraft Equipage on response latencies approached significance, $F(2,30)=3.22, p=.054$. Pilots took more time to respond to workload probes $(M=4.08 \mathrm{sec})$ in the ACARS condition 
than the Voice $(M=3.74 \mathrm{sec}, p=.058)$ condition. The FANS $(M=3.87 \mathrm{sec})$ condition was not different from the other two conditions. There was no effect of Pilot Role on response latencies, $(F<1)$.

Timeouts, or when a participant failed to respond to a workload probe within the 30 -second response window (presumably because workload was too high to attend to the probe) occurred $8.4 \%$ of the time. Pilots timed out $9.8 \%$ of the in the ACARS condition compared to $7.7 \%$ in both Voice and FANS conditions. A repeatedmeasures ANOVA of timeouts as a function of Pilot Role and Aircraft Equipage found a significant effect of Aircraft Equipage $(F(1.27,18.98)=4.19, p<.05)$ but no effect of Pilot Role $(F(1,15)=2.92, p=.11)$.

Pilot probe workload ratings ( 1 bored to 5 busy) were then averaged by crew. Probe workload ratings as a function of Aircraft Equipage showed no significant effects, $F(2,14)=1.57, p=.24$. Overall, pilots found the workload of the simulated flights $(M=2.17)$ one rating higher than boring.

Table 1. Means \& Standard Deviations of Post-Trial Crew Workload and Acceptability Ratings by Aircraft Equipage

\begin{tabular}{lccc}
\hline Question & FANS & ACARS & Voice \\
\hline Overall workload associated with your flight & $2.24(.21)$ & $2.47(.24)_{\mathrm{a}}$ & $2.12(.17)_{\mathrm{a}}$ \\
Peak workload associated with your flight & $2.51(.37)$ & $2.79(.48)^{*}$ & $2.36(.35)^{*}$ \\
Overall workload associated with avoiding weather & $2.16(.16)$ & $2.30(.14)^{*}$ & $2.13(.16)^{*}$ \\
Peak workload associated with avoiding weather & $2.27(.26)$ & $2.47(.33)$ & $2.20(.22)$ \\
Ability to communicate with ATC & $3.95(.35)_{\mathrm{a}}$ & $3.50(.42)_{\mathrm{a}}$ & $4.43(.35)_{\mathrm{a}}$ \\
Ability of ATC to communicate with you & $3.94(.37)_{\mathrm{a}}^{*}$ & $3.59(.46)_{\mathrm{b}}^{*}$ & $4.34(.38)_{\mathrm{ab}}$ \\
Ability to understand what ATC proposed & $4.02(.39)_{\mathrm{a}}$ & $3.66(.49)_{\mathrm{a}}$ & $4.42(.37)_{\mathrm{a}}$ \\
Safety of the flight path you flew & $4.20(.38)$ & $4.05(.49)^{*}$ & $4.41(.35)^{*}$ \\
Efficiency of the flight path you flew & $3.90(.41)$ & $3.76(.51)$ & $4.03(.53)$ \\
\hline
\end{tabular}

Note. Different subscripts represent means that are significantly different at $p<.05$. Asterisks represent means that are different at $p<.07$, but not at $p<.05$.

\subsection{Ratings}

At the end of each trial, pilots provided five acceptability ratings ( 1 not acceptable to 5 excellent) related to communication with ATC and safety and efficiency of their flight path (see Table 1). Voice was rated the highest, FANS next highest and ACARS lowest in ability to communicate with $\operatorname{ATC}(F(2,14)=38.67, p<.01)$, ability of ATC to communicate with them $(F(2,14)=18.98, p<.01)$ and ability to understand what ATC proposed $(F(2,14)=34.51, p<.01)$.

A similar pattern also emerged when crews rated the safety $(F(2,14)=6.18, p<$ .05 ; and the efficiency of the flight path flown $(F(2,14)=2.35, p=.13)$. Crews found that across all conditions, flight path safety was good $(M=4.22)$ and efficiency was marginally good $(M=3.90)$. 


\subsection{Post Simulation Ratings and Comments}

After the simulation, pilots rated their agreement with 15 statements (1 strongly disagree to 5 strongly agree) about each of the different communication procedures. These questions pertained to crew coordination, air-ground communication, safety and acceptability of trajectory based operations, time required for condition specific procedures, workload, and overall concept acceptance. Data were analyzed by pilot with multiple ANOVAs. Twelve of the 15 statements directly compared the three datacom conditions. For all 12 of these statements, the mean preference ratings was lowest for ACARS and highest for Voice, with FANS falling in the middle (all statistically significant at the $p<.05$ level). Given that ACARS and FANS procedures were the least familiar to the pilots, this finding is unsurprising. Pilots prefer familiar procedures. Additionally, ACARS flight requests required significantly more work and thus were received the least well.

Pilots also provided open-ended feedback regarding each communication condition. Pilots had three major concerns about the datacom procedures. The first, concern was ATC response time (mentioned by 7/16 pilots for FANS and 13/16 for ACARS). In current-day operations with voice, the controller responds to messages as they are received. However, in a datacom environment, they seem to respond to messages based on traffic management needs. For example, an aircraft close to being handed off may have been responded to before an aircraft in the middle of the sector with a previous request.

A second concern of pilots was the time and effort needed to create and manage messages on the CDU (mentioned by $7 / 16$ for both FANS and ACARS). Finally, there were other concerns related to message format, such as how the ACARS clearance was formatted and how the ACARS and FANS messages came up across two pages, thereby requiring additional effort to integrate.

\section{Conclusion}

The move to TBO, central to NextGen, has been hampered by low FANS equipage levels in the current fleet. Air carriers are reluctant to invest in FANS equipage while such equipage is of little use in the continental United States. At the same time, service providers see little benefit of TBO as long as equipage levels prevent the vast majority of users from operating under those rules. The proposed procedures point to a potential way out of this impasse by providing a path to TBO that does not require substantial levels of FANS equipage.

While other studies have shown clear benefits to datacom procedures, our results show a more mixed picture. In particular, pilots show a strong preference for Voice. Why the difference? Our study stressed pilot requests, while previous work has looked almost entirely at clearances initiated by the controller. Datacom equipment found on current-day transport category aircraft makes it difficult to formulate 
requests and lack the immediate feedback of traditional voice protocols. The generally slow response time for datacom has been noted in other studies [9, 10]. Groce and Boucek [11] specifically note that pilots found datacom unacceptable for weather avoidance clearances because of the time critical nature of such clearances.

It is possible that a mix of datacom and voice could result in more acceptable response times while accruing many of the benefits of datacom (such as reduced transmission error, the ability to transmit more complex clearances and a reduction in voice traffic). For example, pilots could make requests by voice and receive an acknowledgement by voice which would then be followed up by a clearance. Several pilots in our study stated during the debriefing that their concerns about datacom would be greatly ameliorated if requests were acknowledged more promptly even if there was a delay in the actual response.

Acknowledgement. This study was supported by the NASA Airspace Program, Concepts and Development Project. We would like to thank George Lawton, Dominic Wong, Riva Canton, Tom Quinonez and John Luk for software programming support, and Sarah Ligda and Patrick Cravalho for assistance with recruiting participants. We would particularly like to thank Dr. Kim Vu and Dr. Tom Strybel of California State University, Long Beach, as well for their aid and support in running this study.

\section{References}

1. Joint Planning and Development Office: Concepts of Operations for the Next Generation Air Transportation System, Version 3.0, Washington, DC (2010)

2. Kerns, K.: Air-Traffic Control/Flight Deck Integration. In: Wise, J.A., Hopkin, V.D., Garland, D.J. (eds.) Handbook of Aviation Human Factors, 2nd edn., pp. 23-1-23-17. CRC Press, Boca Raton (2010)

3. McGann, A., Morrow, D., Rodvold, M., Mackintosh, M.-A.: Mixed-Media Communications On The Flight Deck: A Comparison Of Voice, Data Link, And Mixed ATC Environments. Int. J. Aviat. Psychol. 8, 137-156 (1998)

4. Tallota, N.J.: Controller Evaluation Of Initial Data Link Terminal Air Traffic Control Services: Mini-Study 3, vol. I, Report No. DOT/FAA/CT-92/18, I. US Department of Transportation, Federal Aviation Administration, Washington, DC (1992)

5. Mueller, E.: Experimental Evaluation Of An Integrated Datalink And Automation-Based Strategic Trajectory Concept. In: Proceeding of the 7th American Institute of Aeronautics and Astronautics (AIAA) Aviation Technology, Belfast, Northern Ireland (2007)

6. Mueller, E., Lozito, S.: Flight Deck Procedural Guidelines for Datalink Trajectory Negotiation. In: Proceedings of the 8th American Institute of Aeronautics and Astronautics (AIAA) Aviation Technology, Integration, and Operations (ATIO) Conference, Anchorage, AK (2008)

7. Prevot, T.: Exploring The Many Perspectives Of Distributed Air Traffic Management: The Multi Aircraft Control System MACS. In: International Conference on Human-Computer Interaction in Aeronautics, pp. 23-25 (2002) 
8. Granada, S., Dao, A.Q., Wong, D., Johnson, W.W., Battiste, V.: Development And Integration Of A Human Centered Volumetric Cockpit Display For Distributed Air Ground Operations. In: Proceedings of the 12th International Symposium on Aviation Psychology, Oklahoma City, OK, pp. 229-284 (2005)

9. Kerns, K.: Data-Link Communication Between Controllers And Pilots: A Review And Synthesis Of The Simulation Literature. Int. J. of Av. Psych. 1, 181-204 (1991)

10. Smith, N.M., Lee, P.U., Prevot, T., Mercer, J., Palmer, E., Battiste, V., Johnson, W.: A Human-In-The-Loop Evaluation Of Air-Ground Trajectory Negotiation. In: Proceedings of the 4th American Institute of Aerospace and Astronautics Aviation Technology, Integration and Operations Conference, Integration and Operations Conference, Chicago (2004)

11. Groce, J., Boucek, G.: Air Transport Crew Taskin. In: An ATC Data Link Environment. SAE Tech Paper Series, Warrendalte, PA (1987), ISSN 0148-7191 